\title{
Thermal behavior induced by vacuum polarization on causal horizons in comparison with the standard heat bath formalism*
}

Dedicated to A. A. Belavin on the occasion of his 60 th birthday

\author{
Bert Schroer \\ present address: CBPF, Rua Dr. Xavier Sigaud 150, \\ 22290-180 Rio de Janeiro, Brazil \\ email schroer@cbpf.br \\ permanent address: Institut für Theoretische Physik \\ FU-Berlin, Arnimallee 14, 14195 Berlin, Germany
}

Jannuray 2003

\begin{abstract}
Modular theory of operator algebras and the associated KMS property are used to obtain a unified description for the thermal aspects of the standard heat bath situation and those caused by quantum vacuum fluctuations from localization. An algebraic variant of lightfront holography reveals that the vacuum polarization on wedge horizons is compressed into the lightray direction. Their absence in the transverse direction is the prerequisite to an area (generalized Bekenstein-) behavior of entropy-like measures which reveal the loss of purity due to restrictions to wedges and their horizons. Besides the well-known fact that localization-induced (generalized Hawking-) temperature is fixed by the geometric aspects, this area behavior (versus the standard volume dependence) constitutes the main difference between localization-caused and standard thermal behavior.
\end{abstract}

\section{Introductory remarks}

In contradistinction to the standard Boltzmann-Gibbs stochastic framework ${ }^{1}$ of ensembles coupled to a heat bath, which originated in the setting of classical

\footnotetext{
*To appear in the proceedings of the "6th International Workshop on Conformal Field Theory and Integrable Models", in Chernogolovka, September 2002

${ }^{1}$ For brevity the standard thermal setting will be referred to as the "heat bath" formalism, as opposed to thermal manifestations of "localization" whose explanation and investigation constitutes the main topic of this lecture.
} 
physics, the recognition that QFT via its characteristic vacuum polarization effects caused by localization may lead to purely quantum thermal manifestations (even if the global state is a ground state i.e. not a member of an ensemble) is a more recent theoretical discovery which started with Hawking's observation on black hole radiation [1. Although this observation in its original form was dependent on some classical geometrical prerequisites in particular on nontrivial spacetime curvature leading to a Schwarzschild-like black hole of finite radius, it became gradually clear that this thermal manifestation was really a characteristic property for local quantum physics since it is potentially present in all quantum theories (different from quantum mechanics) with a built-in causal propagation structure as a result of a maximal propagation velocity. It is not even limited to the kind of field theoretic models used in relativistic particle physics, but extends to black hole" analog quantum systems [2] in acoustics (phonon excitations), quantum optics or hydrodynamics; in short to all quantum system which admit a Fock space structure with causally propagating quantum excitations. Although the mathematical manifestation of thermal properties in such a local quantum physics setting is omnipresent (see the Tomita-Takesaki theorem in conjunction with the quantum field theoretic Reeh-Schlieder property in the next section), it is not so easy to find conditions under which it leads to observable physical consequences since (at least on the present level of understanding) physical manifestations require very special geometric circumstances. A prime example, which also will serve as our main illustration, is the Gedanken experiment of Unruh 3 in which the causal horizon of a Rindler wedge region in Minkowski spacetime is realized with the help of a family of uniformly accelerated observers (whose worldlines stay inside a wedge such that the (upper) horizon of the wedge defines a "causal protection" against any backreaction of the outside world). In Minkowski spacetime this wedge situation is the only region which admits a geometrical symmetry (a diffeomorphism associated with a Killing vector) which induces an automorphism of the algebra of observables which is generated by local fields associated with the chosen localization region (in conformal invariant theories the modular group of double cone algebras is also geometric [4])

At the time of Hawking's and Unruh's discovery there was an unrelated general structural observation within QFT made by Bisognano and Wichmann [4. Its main point consisted in demonstrating that the application of the TomitaTakesaki modular theory [5] 6 ] to the operator algebra $\mathcal{A}(W)$ generated by fields smeared with test functions with support in the wedge leads to a physical identification of the modular objects associated with the pair $(\mathcal{A}(W), \Omega)$ with $\Omega$ being the vacuum state vector. The modular group turned out to be the wedge associated Lorentz boost and the Tomita involution became identified (up to spatial rotation) with the all important TCP operation. The T-T modular theory was a significant mathematical conquest made one decade before, whose physical significance for the conceptual basis of infinite volume quantum statistical mechanics was a completely independent seminal contribution of Haag, 
Hugenholz and Winnink ${ }^{2}$. The new aspect of the Bisognano-Wichmann observation was that the KMS property, which, as the $\mathrm{H}-\mathrm{H}-\mathrm{W}$ paper had shown, was characteristic for equilibrium thermal quantum physics in the thermodynamic limit (i.e. when the Gibbs state formulation looses its mathematical meaning), does become (via modular theory) also an attribute of the wedge-restricted vacuum state. Different from the standard heat bath Gibbs ensemble formalism, the temperature of the restricted vacuum state (which thermalizes through its restriction from the global algebra to the wedge localized subalgebra) is not a free parameter but, as in Hawking's black hole case or in Unruh's illustration, is determined by the geometry of localization. It did not take long before the deep connection of the Hawking-Unruh aspect with the KMS property of localization as observed by Bisognano and Wichmann was made [17; this was followed by a steady stream of papers in which the relation of thermal aspects of quantum matter with their geometrical localization behind causal horizons ${ }^{3}$ was investigated (see the extensive list of reference in [6.

The aim of these notes is to review the role of the KMS property and modular theory as the unifying formalism behind both the conventional heat bathand the more subtle localization- induced thermal properties, for an extensive article with a similar agenda see [7]. Recent studies [8] have shown that further insight into thermal aspects of localized matter can be obtained by using an algebraic adaptation of the idea of holographic 9 projection. The holographic projection of a wedge algebra consists of an algebra on its (upper) lightfront horizon (half of the lightfront which linearly extends the wedge horizon). Although the global algebra of the lightfront is equal to that of ambient Minkowski space and (in theories with causal propagation) this equality continues to hold between the wedge algebra and that of its horizon, the local structure (the inclusion-preserving system of local subalgebras) of the holographic projection brings to light an interesting correlation structure which the net structure of the wedge algebra does not reveal. Namely the vacuum which (in absence of external fields) suffers vacuum polarization in all space- time and light-like direction presents itself with respect to the lightfront degrees of freedom as a state without transverse vacuum polarization; the omnipresent field theoretic vacuum polarizations have been compressed into the (longitudinal) lightray direction in the process of holographic projection in such a way that the transverse direction remains completely free of vacuum fluctuations (in the standard lightfront quantization methods these important but subtle properties are easily overlooked [10]). One can then check that this somewhat unexpected partial return to vacuum polarization-free quantum mechanics in the transverse direction is physically backed up by the appearance of transverse Galilei invari-

\footnotetext{
${ }^{2}$ One of the important observations in the HHW paper was that the Kubo-MartinSchwinger condition (which was proposed as an analytic trick to avoid the computation of traces) played a fundamental conceptual role in the formulation of statistical mechanics of open systems and (as was later shown in based on observations in) for the notion of stability of equilibrium states 4 .

${ }^{3}$ The event horizons related to Killing vectors in GR are causal horizons, but the latter (which arise as boundaries after causal completion of arbitrary spacetime regions) are not restricted to Killing symmetries (example: double cones in Minkowski spacetime).
} 
ance which originates from the lightfront holography of the translational part of Wigner's "little group" [8]. This invariance subgroup of the lightray forms a two-parametric subgroup of the seven-parametric automorphism group of the lightfront which arises as the subgroup of the ten-parametric Poincaré group, but there are also new geometric invariances (the diffeomorphism of chiral theories) which are invisible before the holomorphic projection because they are "fuzzy" symmetries of the massive theory in the ambient spacetime structure.

The lightfront holography illustrates in greater detail that the crucial property, by which localization in local quantum physics leads to thermal properties, is the appearance of vacuum polarization. Whereas the important role of virtual and real pair creation was appreciated ever since Hawking's famous paper on black hole radiation, the vacuum fluctuation-free transverse tensor structure of the holographic lightfront projection entails an additional interesting consequence. Namely any extensive physical quantity on the horizon which behaves additively if the system is in a special uncoupled state which factorizes under nonoverlapping spatial subdivisions ${ }^{4}$ will be characterized by an area- (transverse volume-) density which can be computed in terms of the longitudinal correlations (which turn out to correspond to a chiral theory). Here the entropy and its quantum description in terms of the von Neumann trace formula based on the density matrix of an impure state immediately comes to ones mind. The problem of localization entropy however requires a bit more abstract setting which also makes perfect sense in the standard heat bath case. One could view the quest for entropy as the search for an additive quantity (in the aforementioned sense) which still would leave open the problem of its normalization. To solve this, one could refer to the way in which this quantity enters the fundamental thermodynamic law, i.e. after having derived the form in which it enters the fundamental laws (which relate it to other quantities) one could fix the normalization by a convention which of course should reduce to the classical case if the fundamental law has the classical form. This is in a way what Bekenstein does when he relates his area proportional entropy to the quantum Hawking theory by invoking the classical form of fundamental thermodynamic law. The main change in adapting the Bekenstein argument to black hole analogs would be on the quantum Hawking side in that a quantity called the "surface gravity" 5 which relates dynamical properties of local quantum physics to the geometric situation (in the more abstract setting of the next section it links the KMS "Hamiltonian" to the modular group) has to be modified according to the physical nature of the analog. According to the best of my knowledge there has been no attempt to derive fundamental thermodynamic laws in case the thermal behavior does not result from an Gibbs-like quantum ensemble average but rather from vacuum fluctuations caused by localization.

The next section is a review of the standard heat bath situation and its relation to the Tomita-Takesaki modular theory and KMS properties 44 [11 12.

\footnotetext{
${ }^{4}$ The vacuum of second quantized interaction-free QM is an example for such a state without entanglement under subdivisions (tensor-product factorization).

${ }^{5}$ In the case of the Unruh Gedanken experiment and black hole analogs this terminology should be taken with a grain of salt.
} 
In the third section we will provide arguments in favor of the existence of an unnormalized entropy which determines ratios of area densities for different quantum matter content. Although one does not expect the totally kinematic situation of Bekenstein, the universality aspects of the holographic projection only permit a dependence on holographic universality classes. The holographic projection reduces the problem of area density to the computation of localization entropy to that in an associated chiral theory.

In order to keep the length of the reference list in balance with the short size of the presentation, I have opted to refer to reviews and books which contain a rather extensive bibliography (including the original papers on the subject) as much as possible. Especially those readers who find these notes too scarce are encouraged to look at those sources.

\section{Heat bath thermal behavior, KMS property and modular theory}

The correlation functions of a QFT in a quantization box V (in order to obtain a discrete energy-momentum spectrum) coupled to a heat bath reservoir are computed with the well-known Gibbs formula

$$
\begin{aligned}
\omega_{\beta}(A) & :=\frac{1}{Z_{V}} \operatorname{tre}^{-\beta H_{V}} A, Z_{V}=\operatorname{tre}^{-\beta H_{V}} \\
& \curvearrowright \omega_{\beta}(\mathbf{1})=1
\end{aligned}
$$

which assigns a (normalized) state $^{6}$ on the algebra of bounded operators. $A \in$ $\mathcal{A}=B(\mathcal{H})$. The Gibbs formula is meaningful as long as the partition function $Z$ exists (which requires a discrete Hamiltonian spectrum bounded below and with finite degeneracy). For this reason the Gibbs formulation starts from a system enclosed in a box. As in the vacuum case one may restrict ones interest to case that the algebras are generated by pointlike fields in which case the operator theory may be reconstructed from the thermal correlation functions of fields and one has access to a perturbative formulation. The difference to the vacuum situation becomes more visible on the level of the operator formalism which may be obtained from the state $\omega_{\beta}(\cdot)$ on $\mathcal{A}(\simeq$ set of correlation functions) by the canonical GNS (Gelfand, Neumark and Segal) construction [4] which by now is well known among mathematical physicist. Using the special property of density matrix states, one may implement the abstract GNS construction on a Hilbert space $\mathcal{H}_{H S}$ whose vectors are Hilbert-Schmidt operators $\kappa$ i.e. $\operatorname{tr} \kappa^{*} \kappa<\infty$

$$
\begin{gathered}
\mathcal{H}_{H S}=\left\{\psi_{\kappa} \mid\left(\psi_{\kappa}, \psi_{\kappa^{\prime}}\right) \equiv \operatorname{tr}^{*} \kappa^{\prime}\right\} \\
\pi(A) \psi_{\kappa} \equiv \psi_{A \kappa} \in \mathcal{H}_{H S}
\end{gathered}
$$

\footnotetext{
${ }^{6}$ The existence of inequivalent representations in the presence of infinitly many degrees of freedom requires to make a distinction between a state in the sense of assigning an expectation value to an operator and a a state vector which implements this state if one goes beyond finite degrees of freedom QM.
} 
where $\pi(\cdot)$ denotes the representation of the algebra on $\mathcal{H}_{H S}$. The HS Hilbert space is isomorphic to the tensor product of the original Hilbert space $\mathcal{H}_{H S} \simeq$ $\mathcal{H} \otimes \mathcal{H}$ since the linear combinations of "dyads" $|\psi\rangle\langle\varphi|$ from the tensor product upon closure in $\mathcal{H}_{H S}$ generate the HS Hilbert space. This "doubling" entails that besides the left action (21) of the full algebra of bounded operators $B(\mathcal{H})$ on $\mathcal{H}_{H S}$ there is a right action which in the HS description reads $\psi_{\kappa} \rightarrow \psi_{\kappa A}$. In order to distinguish between the left and right representation and to maintain the naturality of composition (representation) laws, one defines the right representation as a conjugate (antilinear) representation

$$
\begin{aligned}
\pi_{l}(A) \psi_{\kappa} & =\pi(A) \psi_{\kappa}=\psi_{A \kappa} \\
\pi_{r}(A) \psi_{\kappa} & =\psi_{\kappa A^{*}}
\end{aligned}
$$

It is obvious that any right action commutes with any left action i.e. $\pi_{r}(\mathcal{A}) \subseteq$ $\pi(\mathcal{A})^{\prime}$ (where the dash denotes the von Neumann commutant of $\pi(\mathcal{A})$ in $\mathcal{H}_{H S}$ ) and it is not difficult to see that in fact equality holds [5]. In $\mathcal{H}_{H S} \simeq \mathcal{H} \otimes \mathcal{H}$ there are many more operators than in $\pi(\mathcal{A})$, e.g. the anti-unitary "flip"

$$
\begin{array}{r}
J \psi_{\kappa}:=\psi_{\kappa *}, \quad J^{2}=1 \\
J \pi(A) J=\pi_{r}(A)
\end{array}
$$

which in the tensor product representation would simply interchange the bra and ket in a dyad.

Using now the fact that the Hilbert-Schmidt operator $\kappa_{0} \equiv \rho^{\frac{1}{2}}$ associated with the nondegenerate (no zero eigenvalue) Gibbs density matrix

$$
\omega_{\beta}(A)=\left(\psi_{\kappa_{0}}, \pi(A) \psi_{\kappa_{0}}\right)=\operatorname{tr} \kappa_{0} A \kappa_{0}
$$

is cyclic and separating with respect to the action $\pi(\mathcal{A})$ of the algebra (i.e. sufficiently entangled in $\mathcal{H} \otimes \mathcal{H}$ so that the application of this subalgebra permits to approximate any vector in $\mathcal{H} \otimes \mathcal{H}$ and that it is not possible to annihilate the entangled state with a nonzero operator from $\pi(\mathcal{A})$ ), one checks the validity of the relation (mainly an exercise in the correct application of definitions)

$$
\begin{aligned}
S \pi(A) \psi_{\kappa_{0}} & =\pi(A)^{*} \psi_{\kappa_{0}}, A \in \mathcal{A} \\
\text { where } S & :=J \pi\left(\rho^{\frac{1}{2}}\right) \pi_{r}\left(\rho^{-\frac{1}{2}}\right) \curvearrowright S^{2} \subset 1
\end{aligned}
$$

where the last relation is a notation for the fact that the unbounded ${ }^{7}$ operator $S$ is involutive on its domain. By an additional notational convention one gets this relation into the form where it may be used as a special illustration of a very general operator algebra situation

$$
\begin{aligned}
& H_{\mathrm{mod}} \equiv \pi(H)-\pi_{r}(H) \\
& \Delta^{i t} \equiv e^{-i \beta t H_{\mathrm{mod}}}, S=J \Delta^{\frac{1}{2}} \\
& H_{\mathrm{mod}} \psi_{\kappa_{0}}=0, \Delta^{i t} \psi_{\kappa_{0}}=\psi_{\kappa_{0}} \\
& \Delta^{-i t} \pi(A) \Delta^{i t}=\pi\left(\alpha_{\beta t}(A)\right), \alpha_{t}(A)=A d e^{i H t}(A) \equiv e^{i H t} A e^{-i H t}
\end{aligned}
$$

\footnotetext{
${ }^{7}$ Since $\pi_{r}\left(\rho^{-\frac{1}{2}}\right)$ is unbounded even for Hamiltonians with one-sided unbounded spectrum.
} 
Whereas in vacuum QFT the energy operator $H$ (obtained by integrating the energy density) is the same as the generator of the translation, in the heat bath situation it is the doubled Hamiltonian $H_{\text {mod }}$ which leaves the thermal reference state invariant and generates the translation symmetry of the thermal correlation functions (whereas the energy operator fluctuates in the thermal state and these fluctuations become infinitely big in the thermodynamic limit $\mathrm{V} \rightarrow \infty$ ). The unitary $e^{i t H}$ which represents the time translation in the vacuum representation continues to implement the time automorphism through its adjoint action on the algebra but ceases to be a well defined unitary operator in the GNS Hilbert space associated with the thermodynamic limit where its role is taken over by $e^{i t H_{\text {mod }}}$ (which acts on both $\pi(\mathcal{A})$ and $\pi_{r}(\mathcal{A})=\pi(\mathcal{A})$ ). The following KMS relation follows from the cyclicity property of the trace in the Gibbs formula

$$
\begin{gathered}
\omega_{\beta}\left(\alpha_{t}(A) B\right)=\omega_{\beta}\left(B \alpha_{t+i \beta}(A)\right) \\
\exists F_{A, B}(z), F_{A, B}(t)=\omega_{\beta}\left(B \alpha_{t}(A)\right), F_{A, B}(t+i \beta)=\omega_{\beta}\left(\alpha_{t}(A) B\right)
\end{gathered}
$$

where the second line expresses the analytic content of the KMS condition in more careful terms: there exist an analytic functions $F_{A, B}(z)$ which interpolates between the thermal expectation values of operator products taken in different orders ; this function is analytic in the strip $0<\operatorname{Im} z<\beta$ and has continuous boundary values on both margins which relate to the two different orders according to the above formulas.

There are good reasons in favor of a formulation which deals with infinitely extended system from the very outset (and not as a result of a thermodynamic limiting process on box-enclosed systems) and to consider finite systems as open subsystems [4. These formulations substitute the Gibbs formula (the Gibbs density matrix as a trace class operator on Fock space is only meaningful for box-enclosed systems) by the KMS property, which does not refer to particular representations and trace class operators. In this more general setting heat bath thermal physics becomes incorporated into a mathematical very deep and physically extremely useful theory: the Tomita-Takesaki modular theory. At the heart of that theory is the following theorem [5].

Theorem 1 (main theorem of the Tomita-Takesaki modular theory) Let $(\mathcal{A}, \mathcal{H}, \Omega)$ denote a weakly closed (von Neumann) operator algebra $\mathcal{A}$ acting on a Hilbert space $\mathcal{H}$, with $\Omega \in \mathcal{H}$ a vector on which $\mathcal{A}$ acts in a cyclic and separating manner. Then there exists an antilinear closed involutive operator $S$ which has the dense subspace $\mathcal{A} \Omega$ in its domain such that

$$
\begin{aligned}
S A \Omega & =A^{*} \Omega, A \in \mathcal{A} \\
S & =J \Delta^{\frac{1}{2}}, J \Delta=\Delta^{-1} J
\end{aligned}
$$

The polar decomposition of $S$ leads to an antiunitary $J$ and a positive $\Delta$ which in turn defines the unitary modular group $\Delta^{i t}$. Their significance results from 
their adjoint action on the algebra

$$
\begin{aligned}
J \mathcal{A} J & =\mathcal{A}^{\prime} \\
\sigma_{t}(A) & \equiv \Delta^{i t} A \Delta^{-i t} \in \mathcal{A}
\end{aligned}
$$

The modular automorphism $\sigma_{t}$ fulfills the following KMS property (with $\beta=-1$ )

$$
\omega\left(\sigma_{t}(A) B\right)=\omega\left(B \sigma_{t-i}(A) B\right), \omega(\cdot) \equiv(\Omega, \cdot \Omega)
$$

and depends only on the state $\omega$ (and not on its implementing vector $\Omega$ ).

Any faithful state $\omega$ on an operator algebra leads to this situation via the GNS construction, and any KMS state is faithful [5]. Even though $J$ implements an antiisomorphism of $\mathcal{A}$ with its commutant $\mathcal{A}^{\prime}$ and both together generate the full ambient algebra $B(\mathcal{H})=\mathcal{A} \vee \mathcal{A}^{\prime}$, the validity of a tensor product representation $B(\mathcal{H})=\mathcal{A} \otimes \mathcal{A}^{\prime}$ (with a factorizing modular unitary) is restricted to the case of $\mathcal{A}$ being a type I algebra and $\omega(\cdot)$ is induced by a density matrix (i.e. the KMS property leads back to a Gibbs density matrix). The speciality of the above Gibbs density matrix situation as compared to the general KMS property in the thermodynamic limit is precisely this tensor product structure (which gets lost in the limit when the algebra changes its type to type $\mathrm{III}_{1}$ ). This can be seen explicitly by solving the KMS relation which (for a scalar field) leads to the well known thermal two point function (the higher point functions have the standard free field product structure in terms of the two point function)

$$
\begin{aligned}
& \omega_{\beta}(A(x) A(y))=\frac{1}{(2 \pi)^{3}} \int\left(e^{-i p(x-y)} \frac{1}{1-e^{-\beta \omega}}+e^{i p(x-y)} \frac{e^{-\beta \omega}}{1-e^{-\beta \omega}}\right) \frac{d^{3} p}{2 \omega} \\
& \pi_{\beta}(A(x))=\frac{1}{(2 \pi)^{\frac{3}{2}}} \int\left(e^{-i p x} \pi_{\beta}(a(p))+e^{i p x} \pi_{\beta}(a(p))^{*}\right) \frac{d^{3} p}{2 \omega}, \quad p_{0}=\omega=\sqrt{\vec{p}^{2}+m^{2}} \\
& \left(\begin{array}{cc}
\pi_{\beta}(a(p)) \\
\pi_{\beta}(a(p))^{*}
\end{array}\right) \equiv\left(\begin{array}{cc}
\cosh \alpha(p) & \sinh \alpha(p) \\
\sinh \alpha(p) & \cosh \alpha(p)
\end{array}\right)\left(\begin{array}{c}
a(p) \otimes 1 \\
1 \otimes a^{*}(p)
\end{array}\right), \cosh \alpha(p)=\sqrt{\frac{1}{1-e^{-\beta \omega}}} \\
& \omega_{\beta}(A(x) A(y))=\omega_{0,0}\left(\pi_{\beta}(A(x)) \pi_{\beta}(A(y))\right)
\end{aligned}
$$

where the third line is Bogoliubov transformation on ordinary creation/annihilation operators in a tensor doubled Fock space and the last line states that the thermal two point function can be written as a vacuum representation of the thermal $\pi_{\beta}(A(x))$ operators in a tensor product vacuum ("purification by doubling"). Contrary to the finite volume Gibbs situation the above infinite volume Bogoliubov transformation has no unitary implementation which could undue the entanglement with respect to the doubling associated with the Bogoliubov transformation; in fact these persistent thermal fluctuations in unbounded spacetime are the cause for the loss of the tensor product structure (and the change from type I to type III) between the global von Neumann algebra generated by the fields $\mathcal{A}$ and its commutant $\mathcal{A}^{\prime}$ in the thermal Hilbert space ${ }^{8}$. Not only are the

${ }^{8}$ Whereas the GNS construction on the finite volume (Gibbs) theory leads to a quantum 
thermal infinite volume algebras inequivalent to vacuum algebras, but even the algebras for different temperatures turn out to be inequivalent [5]. Although "purification" of impure states can always be achieved by enlargement, there is no Bogoliubov transformation formula for interacting infinite thermal systems.

A KMS-based direct approach reveals the following additional interesting structural differences to vacuum QFT.

- Unlike vacuum QFT the analytic properties of spacetime or momentum space correlation functions cannot be described in terms of different boundary values one holomorphic "master function"; this complicates in particular the relation between the imaginary time (Matsubara formalism) and real time correlation functions. Even though Lorentz invariance is spontaneously broken, the continued validity of relativistic causality leads to an enlargement of KMS analyticity which was only recently noticed (the relativistic KMS property in [12]).

- Contrary to the vacuum theory where (at least in the case of mass-gaps) the LSZ scattering theory allows a description in the Fock space of the asymptotic particles without encountering infrared divergencies, real time infinite volume thermal theories calculated perturbatively starting from free thermal propagators lead to perturbative infrared problems. Whereas ultraviolet problems in renormalizable theories tend to be of technical origin (cavalier use of singular pointlike fields), difficulties in the infrared invariably point to an insufficient physical understanding. This problem was recently addressed by a concrete proposal on the asymptotic behavior 13. which takes into account the fact that some interaction dependent dissipative effects are asymptotically persistent. This proposal if used in perturbation theory, should avoid infrared problems; a task which still has to be carried out.

Most perturbative calculations have been done in the imaginary time (ITF) Matsubara formalism which by functional formulation relates to the Gibbs setting. In that case (as for spacelike separations), the KMS property simplifies to a periodicity relation which in turn leads to discrete energies. The rather involved relation of ITF with areal time (RTF) formalism (which requires the application of Carlsonian theorems in order to reconstruct the correlations for arbitrary energies from the discrete ITF values) was only recently clarified [16]. For two decades a special formulation of RTF known under the name "Thermo Field Theory" [14 gained increasing popularity with practitioners since it allows to do calculations in terms of generalized ("doubled") Feynman rules with the same efficiency as in vacuum problems. It results from the tensor product doubling of Fock space before the thermodynamic limit; the "Tilde" copy of an

mechanical type $\mathrm{I}_{\infty}$ von Neumann algebra whose characteristic property is the existence of minimal projectors $(\simeq$ maximal measurements), the infinite volume finite temperature QFTs (including [12) are typically of "hyperfinite type $\mathrm{III}_{1}$ ". As will be shown in the next section, localization-caused vacuum fluctuations without a heat bath environment have similar thermal consequences. 
observable in the second tensor factor is simply the Tomita $J$-mirrored operator (for a more detailes about its connection with the older KMS formalism see [15]). Modular theory shows that the tilde fields survive the thermodynamic limit but loose their Fock space tensor product structure relative to the standard fields. Mathematical physicist usually prefer the older KMS formulation [4] since its relates in a stronger and conceptually less mystifying way to physical and mathematical principles. Our preference of KMS in these notes is a result of the fact that localization-caused thermal properties do not arise as thermodynamic limits of Gibbs situations; rather the situation is the other way around in that one has to invent such tensor product interpolations (the "split property" of next section) in order to be able to envisage a localization entropy corresponding to its KMS temperature.

In the next section it will be shown that the modular framework is capable of incorporating both the heat bath- and the localization- caused thermal properties.

\section{Thermal aspects caused by vacuum polariza- tion on horizons}

As mentioned in the introduction, the Hawking black hole thermal aspects are not limited to event horizons generated by spacetime curvature in general relativity, but they occur in all systems (the black hole analogs) of local quantum physics i.e. quantum systems with a notion of causal propagation of quantum matter (and the ensuing inevitable vacuum polarization). In a way this kind of thermalization without invoking heat bath ensembles is related to the loss of information by restricting states to subalgebras. But there is a caveat, according to the Tomita-Takesaki theorem the thermalization (i.e. the KMS property of the subalgebra-restricted state) takes place iff this restriction causes the state vector to be cyclic and separating. Although this does not happen in QM (where the restriction to a compact region would lead to an inside/outside tensor product factorization without any entanglement of the vacuum) in line with the absence of causal propagation with a maximal velocity, this is a vastly general phenomenon in QFT thanks to the omnipresence of vacuum polarization. The Reeh-Schlieder theorem of QFT (the localization generated "operator-state" relation in the more folkloristic terminology often used in conformal QFT) insures that these properties are always fulfilled as long as the localization region has a nontrivial causal disjoint. Although the Tomita-Takesaki theorem asserts that the modular KMS automorphism always exists in these cases, the physical interpretation up to now has been restricted to cases of geometric (diffeomorphism, non-fuzzy) action of the modular group $\sigma_{t}$ which in the context of massive QFT in Minkowski space leaves only the Lorentz boosts of wedge region (and in CST the much richer cases of horizons associated with Killing symmetries). The best known illustration without curvature is Unruh's Gedankenexperiment already mentioned in the introduction in which the observables localized in a wedge re- 
gion (the Bisognano-Wichmann of a ) bounded by a causal horizon are realized by a family of uniformly accelerated observers whose Hamiltonian is proportional to the Lorentz boost (for a simple but enlightening presentation using modular concepts see [17).

This raises the question whether the area behavior of entropy (observed first with the help of classical arguments applied to black hole horizons by Bekenstein) could also be expected to be the manifestation of the same vastly general mechanism which is responsible for the appearance of a temperature via quantum localization. Trying to answer this question with standard box quantization methods and ad hoc cut-offs (to obtain a finite entropy via standard degree of freedom counting) proved to be inconclusive [18. According to the above ideas the relevant question should be whether by physically motivated ideas (i.e. by remaining within the given local theory and thus avoiding ad hoc cut-offs) one can associate a localization entropy with the Lorentz boost in its role as the modular group of the wedge algebra so that the Hawking temperature (which cannot be used to get informations about quantum gravity) and the area density of entropy live under a common roof. Since the upper horizon algebra turns out to be globally identical to the wedge algebra (but has a very different local structure!), this question can be reformulated in terms of the scale transformation to which the boost reduces on the horizon (half the lightfront). The formalism which achieves this reformulation including the determination of the new local structure on the horizon is "algebraic lightfront holography", a mathematically and conceptually rigorous variant of the old lightfront (or $\mathrm{p} \rightarrow \infty$ frame) formalism in which greater attention is payed to the locality of both the original ambient theory and its projection as well as to the fine points of their only partially local mutual relation (The lightfront is not a globally and even not a locally hyperbolic manifold). It turns out that the lightfront holography leads to a QFT with a seven parametric symmetry subgroup of the Poincaré group which contains in particular a transverse Galilei group which results from the holographic projection of the "translations" contained in Wigner's 3-parametric "little group" of the lightray in the lightfront [8]. Limiting the presentation for reasons of pedagogical brevity to free fields ${ }^{9}$ where the holographic projection can be obtained in terms of lightfront restriction of fields without first converting them into algebras, one finds for the Weyl generators associated with a scalar free field $A(x)$

$$
\begin{aligned}
& W(f):=e^{i A(f)}, A(f)=\int A(x) f(x) d^{4} x \\
& W\left(g, f_{\perp}\right) \longrightarrow W_{L F}\left(g, f_{\perp}\right)=e^{i A_{L F}\left(g, f_{\perp}\right)}, A_{L F}\left(g, f_{\perp}\right)=\int a^{*}\left(p_{-}, p_{\perp}\right) g\left(p_{-}\right) f_{\perp}\left(x_{\perp}\right) \frac{d p_{-}}{2\left|p_{-}\right|} d^{2} p_{\perp}+h . c . \\
& \curvearrowright\left\langle W\left(g, f_{\perp}\right) W\left(g^{\prime}, f_{\perp}^{\prime}\right)\right\rangle=\left\langle W\left(g, f_{\perp}\right)\right\rangle\left\langle W\left(g^{\prime}, f_{\perp}^{\prime}\right)\right\rangle \text { if suppf } \cap \operatorname{supp}^{\prime}=\emptyset
\end{aligned}
$$

The second line formulates lightfront restriction on the dense set of wedge sup-

\footnotetext{
${ }^{9}$ For interacting fields with infinite wave function renormalization constants the holographic process requires a conversion into operator algebras; but even though the methods turn out to be quite different, the results on the absence of transverse fluctuations are the same [8].
} 
ported test functions which factorize into a longitudinal and a transverse part [8]. The third line is the statement that holographically projected Weyl generators (and therefore also the algebras they generate) have no transverse fluctuations; the holographic projection compresses all vacuum fluctuations into the lightlike direction. This reduces the problem of horizon-associated entropy to the problem of looking for an area (the area of the edge of the wedge which limits the upper horizon) density of entropy and should be interpreted as the localization entropy of a halfline in a chiral theory ${ }^{10}$. From the time of discovery of infinite vacuum fluctuations on the boundary of sharply localized partial Noether charges by Heisenberg it became clear that the remedy for obtaining well defined partial charge operators is to make the spacetime boundary somewhat "fuzzy" by using smearing functions which smoothly interpolate between one and zero. For the more abstract problem of assigning an entropy to a "partial vacuum" (i.e. the ambient vacuum reduced to the horizon algebra) the analog construction is based on the split property namely the assertion that between two sharply localized (necessarily hyperfinite type $\mathrm{III}_{1}$ ) algebras (where the larger one $\mathcal{A}_{\varepsilon}$ is obtained by extending the smaller by an arbitrarily small " $\varepsilon$-collar") there exist tensor-factorizing type I algebras $\mathcal{N}_{\varepsilon}$ on which the vacuum state becomes a faithful density matrix $\rho_{\varepsilon}$

$$
\begin{aligned}
\mathcal{A} & \subset \mathcal{N}_{\varepsilon} \subset \mathcal{A}_{\varepsilon}, \quad B(\mathcal{H})=\mathcal{N}_{\varepsilon} \otimes \mathcal{N}_{\varepsilon}^{\prime} \\
\omega(N) & =\operatorname{tr}\left(\rho_{\varepsilon} N\right), \quad N \in \mathcal{N}_{\varepsilon}
\end{aligned}
$$

This would bring us back to the Gibbs setting, except that in this case one has no explicit formula for $\rho_{\varepsilon}$ in terms of a Hamiltonian; the existence of $\mathcal{N}_{\varepsilon}$ and the form of $\rho_{\varepsilon}$ is rather determined in terms of more abstract modular concepts. The problem of assigning an entropy to the trace class operator $\rho_{e}$ is not different from that in the heat bath situation; in addition to the trace class property of the density one has to make sure that the $\rho_{\varepsilon}$ operator does not have too many eigenvalues around zero which would prevent the convergence in the von Neumann formula $S\left(\rho_{\varepsilon}\right)=-\operatorname{tr} \rho_{\varepsilon} \ln \rho_{\varepsilon}$.

The transverse symmetry and absense of correlations for the case at hand reduces the area density problem to that of a chiral theory on a lightray, which by a Cayley transform becomes a chiral theory on the circle. Cutting the circle into the two halfs $I$ and its opposite $I^{\prime}$ and separating the ends by a distance $\varepsilon$ so that $I_{\varepsilon} \subset I, I^{\prime} \subset I_{\varepsilon}^{\prime}$ our problem is to compute a "split entropy" $S\left(\rho_{\varepsilon}\right)$ associated with a chiral split inclusion $\mathcal{A}\left(I_{\varepsilon}\right) \subset \mathcal{N}_{\varepsilon} \subset \mathcal{A}(I), \mathcal{B}(\mathcal{H})=\mathcal{N}_{\varepsilon} \otimes \mathcal{N}_{\varepsilon}^{\prime}$. The type $I_{\infty}$ algebra $\mathcal{N}_{\varepsilon}$ approaches for $\varepsilon \rightarrow 0$ the sharply localized halfline algebra $\mathcal{N}_{\varepsilon} \rightarrow \mathcal{A}(I)$ so that the modular group corresponding to $\rho_{\varepsilon}$ approximates the dilatation group which in turn is the holographic lightfront projection of the wedge-affiliated boost. The entropy diverges for $\varepsilon \rightarrow 0$ and it is expected that it behaves universally as $\simeq-\ln \varepsilon$. Since we were not able to supply a proof, we formulate the following universality behavior as a conjecture.

\footnotetext{
${ }^{10}$ I learned from Detlev Buchholz that the problem of localization entropy in a more general setting as the present one had been considered as a very hard problem by algebraic field theorist (see e.g. 19). The present holographic setting is expected to make it more amanable.
} 
Conjecture 2 (universality conjecture) The split localization entropy of the halfline in the sharp localization limit goes as

$$
S_{\varepsilon} \underset{\varepsilon \rightarrow 0}{=}-c \ln \varepsilon
$$

where $c$ depends on the chiral model appearing in the holographic projection (the holographic universality class). Hence the area density of entropy associated with the horizon is only determined by the above considerations up to an overall normalization which is independent on the holographic universality class.

The conjecture is consistent with the observation that the there is no specific reference to gravitational aspects, i.e. the above considerations hold also for the black hole analogs.

The conceptually most poignant way to understand the role of this localization entropy is to associate it with a measure of entanglement of the physical vacuum $\omega$ of the ambient theory relative to the product vacuum $\omega_{P}$ which expresses the division of the lightfront into the wedge horizon and its opposite. After taking the transverse symmetry and absence of correlation into account, the problem becomes one of studying a product state on the two-fold localized chiral algebra $\mathcal{A}\left(I_{\varepsilon}\right) \vee \mathcal{A}(I)^{\prime}$

$$
\omega_{p}\left(A A^{\prime}\right)=\omega(A) \omega\left(A^{\prime}\right), \quad A \in \mathcal{A}\left(I_{\varepsilon}\right), A^{\prime} \in \mathcal{A}(I)^{\prime}
$$

In this formulation (using states instead of implementing vectors) there is no direct dependence on the (arbitrarily chosen) intermediate type $I_{\infty}$ factor ${ }^{11}$ and the question arises whether it is possible to define a kind of relative entanglement of $\omega$ with respect to $\omega_{P}$ on $\mathcal{A}\left(I_{\varepsilon}\right) \vee \mathcal{A}(I)^{\prime}\left(\simeq \mathcal{A}\left(I_{\varepsilon}\right) \otimes \mathcal{A}(I)^{\prime}\right)$ directly in terms of these states without implementing them by vectors (entanglement leads directly to impurity upon restriction of. There exists a variational entropy formula by Kosaki, but unfortunately it was not possible to put it to good use for this kind of problem [19. There is however special implementation of the split isomorphism $\Phi\left(\mathcal{A}\left(I_{\varepsilon}\right) \vee \mathcal{A}(I)^{\prime}\right)=\mathcal{A}\left(I_{\varepsilon}\right) \otimes \mathcal{A}(I)^{\prime}$ in a rather large space in which (at least for interaction-free theories) overlaps of states can be bounded above by absolute values of overlap of states. The calculation involves the application of the socalled flip-trick which resembles the Noether formalism for free currents [20]. The result is that the overlap for an algebra generated by a chiral scalar free field can be written as 8 .

$$
e^{-\frac{1}{2}\langle j(f), j(f)\rangle_{0}} \simeq \varepsilon
$$

Here $j$ is a abelian chiral current and $f$ is a smearing function which is one on $I_{\varepsilon}$, zero on $I$ passes smoothly from one to zero in the two $\varepsilon$ intervals between. The only contribution comes from the $\varepsilon$ regions where the function changes. Choosing a fixed smooth function and doing the limit by scaling the transition region, one finds a vanishing overlap going with the first power in $\varepsilon$. One expects

\footnotetext{
${ }^{11}$ However the normality of the product state on the two-fold localized algebra is equivalent to the existence of an intermediate type $I_{\infty}$ factor [4].
} 
a power type of vanishing in every chiral model. In principle one can compute the entanglement of the vacuum in a suitable tensor product basis (e.g. one defined by applying polynomials of the Fourier coefficients of $j$ to the tensor product vacuum). One would expect that the strength of the entropy is universally logarithmic, but that the numerical factors multiplying $-\ln \varepsilon$ depend on the chiral matter which represents the holographic universality classes of the original quantum matter. The universality conjecture is related to the observation that the vacuum polarization related to the split horizon (which is concentrated to the collar of size $\varepsilon$ ) is not a property of short distance singularities of particular field coordinatizations ${ }^{12}$ but rather an intrinsic property of the split-localized algebra itself. Actually it does not appear difficult to use the same implementation as before in order to obtain an upper bound for the $\varepsilon$-dependent split entropy since a natural discrete basis in the tensor product space can be given in terms of the rotational modes of the chiral free field. We hope to return to this problem.

The missing overall normalization should distinguish analog systems from gravitational black holes (there is no distinction in the above considerations) and this is expected to come about through thermodynamic fundamental laws. It is an open question whether and how such laws can be derived for thermal aspects which are not resulting from the standard heat bath ensemble averaging. One possibility would be that such fundamental thermodynamic laws (through which entropy is related to other quantities computed from local quantum physics) need nonvanishing curvature and compact regions of bifurcations (edges) of horizons, but up to now there seems to be no support for this idea.

Whereas the Bekenstein area law is totally independent of the kind of quantum matter (but depends on the relation of the geometrical modular group to the dynamical time development group which distinguished gravitational situations from black hole analogs), its quantum version may have a holographic universality class dependence. In this sense it may contain slightly more structure about the quantum system than the Hawking temperature of the KMS state.

\section{Concluding remarks}

In this paper I argued that the modular approach allows to unify the understanding of heat bath thermal aspects of quantum physics with those caused by vacuum fluctuations which are the inexorable result of causal localization in QFT (even when the global state is the vacuum).

The problem of the area density of quantum entropy associated with an horizon (without or with curvature) has been in the center of interest for a long time. Ever since Bekenstein proposed his famous area law invoking analogies of black hole physics with classical thermodynamics, many physicists expected that its quantum understanding contains important clues about QG beyond

\footnotetext{
${ }^{12}$ The standard ultraviolet divergencies are caused by the use of (inevitably) singular pointlike field coordinatizations of the local net of algebras.
} 
QFT in CST. The above argument in favor of vacuum fluctuation caused by localization calls for caution about the various speculative ideas which propose to solve this problem by invoking "new physics". Presently a more conservative explanation of the area behavior (on the level of the Hawking-Unruh thermal aspects) which maintains all the principles of QFT but adds new conceptual and mathematical tools cannot be excluded. In the previous section it was argued that the solution of the entropy problem in the setting of QFT depends on a certain universality behavior in the holographic projection as well as the validity of normalizing quantum fundamental thermodynamic laws for localizationinduced thermal behavior, both unproven. In contrast to the many speculative attempts ${ }^{13}$, a clarification along these more conservative lines has the additional advantage that its importance would not be diminished by a negative outcome i.e. a clear-cut argument why the conjectured behavior may be incompatible with the principles underlying QFT would also constitute a major achievement.

A shorter report based on a talk with a similar content can be found under hep-th/0301082

Acknowledgments: I am indebted to Detlev Buchholz for explaining some fine points of his work with Jaques Bros on the correct analog of the LSZ asymptotics in the heat bath setting of local quantum physics.

\section{References}

[1] S.W. Hawking, Commun. Math. Phys. 43, (1975) 199

[2] R. Schuetzhold and W. G. Unruh, Gravity wave analogs of black holes, gr-qc/0205099

[3] W. G. Unruh, Phys. Rev. D 14, (1976) 870

[4] R. Haag, Local Quantum Physics, Springer Verlag (1992)

[5] O. Bratteli and D. W. Robinson, Operator Algebras and Quantum Statistical Mechanics, Vol.1, Springer Verlag, New York 1979

[6] H. J. Borchers, J. Math. Phys. 41, (2000) 3604, for a more extensive version of the same work see ESI preprint 773 (1999) http://www.esi.ac.at

[7] L. Birke and J. Fröhlich, KMS, ETC., math-ph/0204023

[8] B. Schroer, JPA 35, (2002) 9165

B. Schroer, Lightfront holography and area density of entropy associated with localization on wedge-horizons, hep-th/0208113 to be published in IJMPA

\footnotetext{
${ }^{13}$ It would be nice if instead of many computations on this subject one could find some interpretation. Since physics should de-mystify nature, one would like to know: if it is not vacuum polarization (which is behind the Hawking thermalization) due to localization on horizons, then what else is responsible for the area behavior of Bekenstein entropy?
} 
[9] G. 't Hooft, in Salam-Festschrift, A. Ali et al. eds., World Scientific 1993, 284

[10] L. Susskind, J. Math. Phys. 36, (1995) 6377

[11] J. Bros and D. Buchholz, Annals Poincaré Phys. Theor. 64, (1996) 495

[12] J. Bros and D. Buchholz, Nucl. Phys. B 429, (1994) 291

[13] J. Bros and D. Buchholz, Nucl. Phys. B 627, (2002) 219

[14] Y. Takahasi and H. Umezawa in Collect.Phenom.2, (1975) 55

[15] I. Ojima, Annals Phys. 137, (1981) 1

[16] G. Cuniberti, E. De Micheli and G. A. Viano, Commun. Math. Phys. 216, (2001) 59

[17] G. L. Sewell, Ann. Phys. 141, (1982) 291

[18] M. Bombelli, R.K Kaul, J. Lee and R. D. Sorkin, Phys Rev. D 34, (1986) 373

[19] H. Narnhofer, in The State of Matter, ed. by M. Aizenman and H. Araki (Wold-Scientific, Singapore) 1994

[20] C. D'Antoni, S. Doplicher, K. Fredenhagen and R. Longo, Commun.Math. Phys. 110, (1987) 325 